\title{
Surfactant-mediated matrix-assisted laser desorption/ ionization time-of-flight mass spectrometry of small molecules
}

\author{
David C. Grant and Robert J. Helleur* \\ Department of Chemistry, Memorial University of Newfoundland, St. John's, NL, A1B 3X7, Canada \\ Received 26 September 2006; Revised 20 December 2006; Accepted 22 December 2006
}

\begin{abstract}
A variety of surfactants have been tested as matrix-ion suppressors for the analysis of small molecules by matrix-assisted laser desorption/ionization time-of flight mass spectrometry. Their addition to the common matrix $\alpha$-cyano-4-hydroxycinnamic acid (CHCA) greatly reduces the presence of matrixrelated ions when added at the appropriate mole ratio of CHCA/surfactant, while still allowing the analyte signal to be observed. A range of cationic quaternary ammonium surfactants, as well as a neutral and anionic surfactant, was tested for the analysis of phenolics, phenolic acids, peptides and caffeine. It was found that the cationic surfactants, particularly cetyltrimethylammonium bromide (CTAB), were suitable for the analysis of acidic analytes. The anionic surfactant, sodium dodecyl sulfate, showed promise for peptide analysis. For trialanine, the detection limit was observed to be in the 100 femtomole range. The final matrix/surfactant mole ratio was a critical parameter for matrix ion suppression and resulting intensity of analyte signal. It was also found that the mass resolution of analytes was improved by $25-75 \%$. Depth profiling of sample spots, by varying the number of laser shots, revealed that the surfactants tend to migrate toward the top of the droplet during crystallization, and that it is likely that the analyte is also enriched in this surface region. Here, higher analyte/surfactant concentration would reduce matrix-matrix interactions (known to be a source of matrix-derived ions). Copyright (C) 2007 John Wiley \& Sons, Ltd.
\end{abstract}

Matrix-assisted laser desorption/ionization (MALDI) is a powerful technique that was first described by Hillenkamp and Karas ${ }^{1}$ and by Tanaka et al. ${ }^{2}$ in the late $1980 \mathrm{~s}$. MALDI evolved from similar desorption/ionization methods such as fast-atom bombardment (FAB) and laser desorption/ionization (LDI) mass spectrometry and it has been found useful in the analysis of macromolecules, such as proteins, oligonucleotides, and synthetic polymers. ${ }^{1-6}$ Its distinguishing feature is that the analyte is embedded in a molar excess of chemical matrix.

Although MALDI time-of-flight mass spectrometry (TOFMS) has proven to be useful in the analysis of macromolecules, its applications to small molecules ( $<500 \mathrm{Da})$ has yet to be fully exploited. One of the fundamental reasons for this has been the abundance of matrix-related ions, due to the decomposition and various reactions of the associated matrix, in the low mass range of spectra.

Several methods have been studied to reduce matrix-ion interference. These include using fullerenes, ${ }^{7,8}$ inorganic compounds $^{2,9-12}$ and high-mass molecules. ${ }^{13,14}$ Carbon nanotubes were first investigated as a potential matrix for MALDI by $\mathrm{Xu}$ et al., ${ }^{15}$ and other studies have looked at

*Correspondence to: R. J. Helleur, Department of Chemistry, Memorial University of Newfoundland, St. John's, NL, A1B 3X7, Canada.

E-mail: rhelleur@mun.ca

Contract/grant sponsor: The Department of Chemistry and the School of Graduate Studies (Memorial University) and NSERC. derivatizing nanotubes and using them as a matrix for cyclodextrins, peptides, carbohydrates and small molecules. ${ }^{16-19}$ Although these methods work well, they have limited application, do not incorporate the conventional, well-established organic matrices, and many of the above materials are not commercially available. Thus, when using commercial organic matrices, techniques for the suppression of matrix-ion signals are still desired to improve the MALDI analysis of small molecules.

Ion suppression was first reported by Chan et al., ${ }^{20}$ who observed an absence of matrix ions when an optimal molar ratio of nicotinic acid to insulin was used. Knochenmuss et al..$^{21}$ further investigated the mole ratio dependent matrix suppression effect (MSE) for small to medium sized analytes (1000-20000 Da) and found that at appropriate matrix/ analyte mixing ratios, the positively charged matrix-related ions could be fully suppressed. This was found to be true regardless of the analyte form, whether it be a radical cation, protonated molecule or alkali-metal adduct. This approach has also been the focus of other studies. ${ }^{22,23}$ Using conventional matrices such as DHB (2,5-dihydroxybenzoic acid) and CHCA ( $\alpha$-cyano-4-hydroxycinnamic acid), a matrix/analyte mole ratio of $<200: 1$ was efficient for large molecule analysis while, for smaller molecules, ratios of $<10: 1$ were selected. It is believed that under these conditions (*)WILEY InterScience 
neutral analytes can deplete primary matrix ions by secondary ion-molecule reactions and when enough analyte is present to react with all excited matrix ions, matrix-matrix reactions will be minimized. Further requirements were that enough analyte must be present and that the laser intensity should not be too high above the intensity threshold.

Another method used to achieve matrix-ion suppression was reported by Guo et al. ${ }^{24}$ They found that the surfactant cetyltrimethylammonium bromide (CTAB) suppressed CHCA-related signals for the analysis of various peptides, cyclodextrin derivatives, and small drug molecules using a matrix/CTAB mole ratio of 1000:1. To our knowledge, they were the first to term this technique 'matrix-suppressed laser desorption/ionization' (MSLDI). This is interesting as surfactants are often avoided in mass spectrometry as they are known to be analyte ion suppressors; however, in this study, the amount of surfactant used was very low. Recently, $\mathrm{Su}$ et al. used this technique to analyze suspect tablets for drugs such as amphetamines and related compounds. ${ }^{25}$

This paper presents a more thorough exploration of the use of $C T A B$ as a matrix suppressor. A variety of other surfactants were also examined to determine if they also induce matrix-ion suppression. In addition, a wider class of small molecule analytes has been investigated with respect to their suitability for use in analysis by surfactant-mediated MALDI-TOFMS including, for the first time, acidic organics such as phenolics and phenolic acids. The optimum ratio of matrix/surfactant has been found for each analyte/surfactant group. Depth profile analysis within a given spot sheds some light on the mechanism of matrix-ion suppression and the properties of the surfactant.

\section{EXPERIMENTAL}

\section{Chemicals}

$\alpha$-Cyano-4-hydroxycinnamic acid (CHCA), $p$-coumaric acid, chrysin, trialanine (Ala-Ala-Ala), caffeine and quercetin were purchased from Sigma (St. Louis, MO, USA). Cetyltrimethylammonium bromide (CTAB), hexyltrimethylammonium bromide (HTAB), dodecyltrimethylammonium bromide (DDTAB), tetrabutylammonium bromide (TBAB), decamethonium bromide (DMB), sodium dodecyl sulfate (SDS) and Brij ${ }^{\mathrm{R}} 30$ were obtained from Aldrich (Mississauga, ON, Canada). Deionized water, methanol and acetone were all HPLC grade purchased from Fisher Scientific (Fair Lawn, NJ, USA). All chemicals were used without further purification.

\section{Sample preparation}

CHCA stock solution was prepared fresh daily at a concentration of $10 \mathrm{mg} \mathrm{mL}^{-1}(52.9 \mathrm{mM})$ in a solution with a 1:4 volumetric ratio of water to methanol. All analytes were initially prepared as $2.65 \mathrm{mM}$ solutions in acetone, to ensure dissolution, and later diluted tenfold in the same solvent as CHCA solutions. Stock solutions of surfactants were also prepared at $2.65 \mathrm{mM}$ in 80:20 methanol/water. When not in use, solutions were stored at $4^{\circ} \mathrm{C}$. Fresh solutions of analytes and surfactants were prepared weekly.

Analytes $(0.265 \mathrm{mM})$ were mixed with matrix at a $1: 1$ volume ratio $(10 \mu \mathrm{L}$ of each) in a plastic centrifuge vial, and
$0.2 \mu \mathrm{L}$ aliquots of various concentrations of surfactant were added so that mole ratios of matrix/analyte/surfactant (M/A/S) of 1000:5:S were achieved, where $S$ ranged from 1 to $1-10^{-5}$. All samples were vortex mixed for $30 \mathrm{~s}$, then centrifuged at $3000 \mathrm{rpm}$ for $30 \mathrm{~s}$ prior to spotting. For MALDI analysis $0.5 \mu \mathrm{L}$ spots were placed on a $96 \times 2$ well MALDI plate (Applied Biosystems, Framingham, MA, USA). The plate had a bonded hydrophobic surface with pre-punched holes with a diameter of $1.3 \mathrm{~mm}$. This spotting surface is notably smaller than a regular MALDI plate $(2 \mathrm{~mm})$ and the hydrophobic perimeter helped produce a spot with more uniform thickness. It should be noted that samples containing surfactant required about $30 \mathrm{~min}$ to crystallize in a desiccator before being loaded into the MALDI-TOF mass spectrometer.

Quercetin and chrysin (phenolics), trialanine (peptide), p-coumaric acid (phenolic acid), and caffeine (an alkaloid) were tested separately with each of the seven surfactants; five basic quaternary ammonium surfactants (HTAB, DDTAB, $\mathrm{CTAB}, \mathrm{TBAB}$ and $\mathrm{DMB}$ ), one anionic surfactant (SDS) and one neutral surfactant $\left(\right.$ Brij $^{\mathrm{B}} 30$ ) (Fig. 1). Each surfactant was mixed into a fixed mole ratio matrix/analyte solution (1000:5) at varying surfactant mole ratios to examine the effect of both the chemical nature of the surfactant and its concentration on the resulting analyte signal. Parameters monitored were the analyte signal-to-noise $(\mathrm{S} / \mathrm{N})$ ratio, resolution and ion signal intensities.
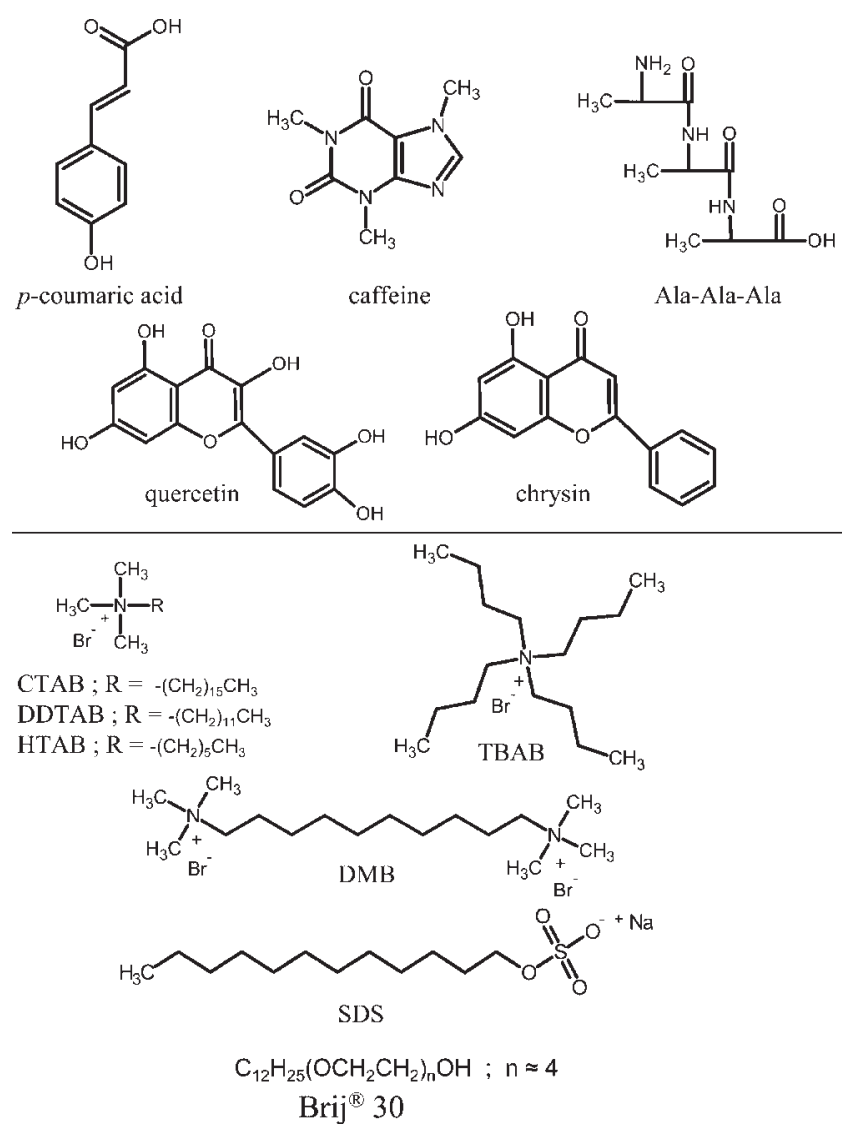

Figure 1. Molecular structures of analytes (top) and surfactants (bottom) used.

Rapid Commun. Mass Spectrom. 2007; 21: 837-845 


\section{Instrumentation}

The MALDI-TOF mass spectrometer was a Voyager $\mathrm{DE}^{\mathrm{TM}}$-PRO from Applied Biosystems (Framingham, MA, USA). The instrument was equipped with a video camera and the sample image was displayed on a monitor enabling the laser to be focused on a given spot and controlled manually. The positive ion reflectron mode was used. The instrument was equipped with a pulsed nitrogen laser ( $337 \mathrm{~nm}, 3 \mathrm{~ns}$ pulse duration, $3 \mathrm{~Hz}$ frequency) and a delayed extraction source. An accelerating voltage of $20 \mathrm{kV}$ and a grid voltage setting of $71 \%$ were used. The laser fluence was set to 2400 arbitrary units and an extraction delay time of $145 \mathrm{~ns}$ was used. The acquisition mass range was $m / z 100-500$ and all spectra were obtained by averaging 25 laser shots, unless otherwise stated. Spectra were analyzed using version 4 of Data Explorer ${ }^{\mathrm{TM}}$ software. All resolution values were calculated at $50 \%$ of the maximum peak height.

\section{RESULTS AND DISCUSSION}

\section{Influence of various surfactants}

Figure 2(a) displays a MALDI-TOF mass spectrum of the CHCA matrix only. As can be seen, there are many ions that are typical of CHCA, such as a protonated molecule $[\mathrm{M}+\mathrm{H}]^{+} m / z$ 190, a sodiated adduct $[\mathrm{M}+\mathrm{Na}]^{+} m / z 212$, and a protonated dimer $[2 \mathrm{M}+\mathrm{H}]^{+} m / z$ 379. These and other matrix-related ions present in the MALDI spectra are listed in Table 1 and agree with other reports. ${ }^{17,18}$ These are low-mass ions, all less than $m / z$ 500, and they can complicate the analysis of small molecules.

Figure 2(b) shows the mass spectrum of CHCA with the addition of the cationic surfactant HTAB at a 1000:1 mole ratio of matrix/surfactant. The only ion observed is from the surfactant, corresponding to $[\mathrm{HTAB}-\mathrm{Br}]^{+} m / z$ 144. All matrix-related peaks are suppressed. Figure 2(c) shows the result of decreasing the mole ratio 100-fold to 1000:0.01. The surfactant-related ion at $m / z 144$ is still dominant, but one of the matrix fragment ions $(m / z 172)$ can be observed. When the ratio is finally lowered to 1000:0.0001, as seen in Fig. 2(d), matrix-ion suppression is lost and major CHCA ions listed in Table 1 are apparent.

The ability of other surfactants to suppress the generation of CHCA-related ions, while allowing the analyte ion signal to be observed, was of major interest; this phenomenon was tested individually for the analysis of five analyte molecules using each surfactant (at various concentrations) and the results are shown in Table 2. Representative of the results, Fig. 3(a) shows a typical MALDI-TOF mass spectrum of $p$-coumaric acid (A) in the absence of surfactant. Two ions corresponding to the analyte are observed, a fragment ion $\left[\mathrm{A}+\mathrm{H}-\mathrm{H}_{2} \mathrm{O}\right]^{+} m / z \quad 147$ and the protonated molecule $[\mathrm{A}+\mathrm{H}]^{+} m / z$ 165. However, these analyte ions are accompanied by an abundance of matrix ions, demonstrating that it would be quite difficult to identify or measure the ion signals of unknown low-mass compounds. Figure 3(b) shows the mass spectrum of the same analyte (A), but with the addition of CTAB (S) to the matrix $(\mathrm{M})$ so that the $\mathrm{M} / \mathrm{A} / \mathrm{S}$ mole ratio is 1000:5:0.1. In this spectrum the analyte signals observed in Fig. 3(a) are still present, but matrix-related ions have been
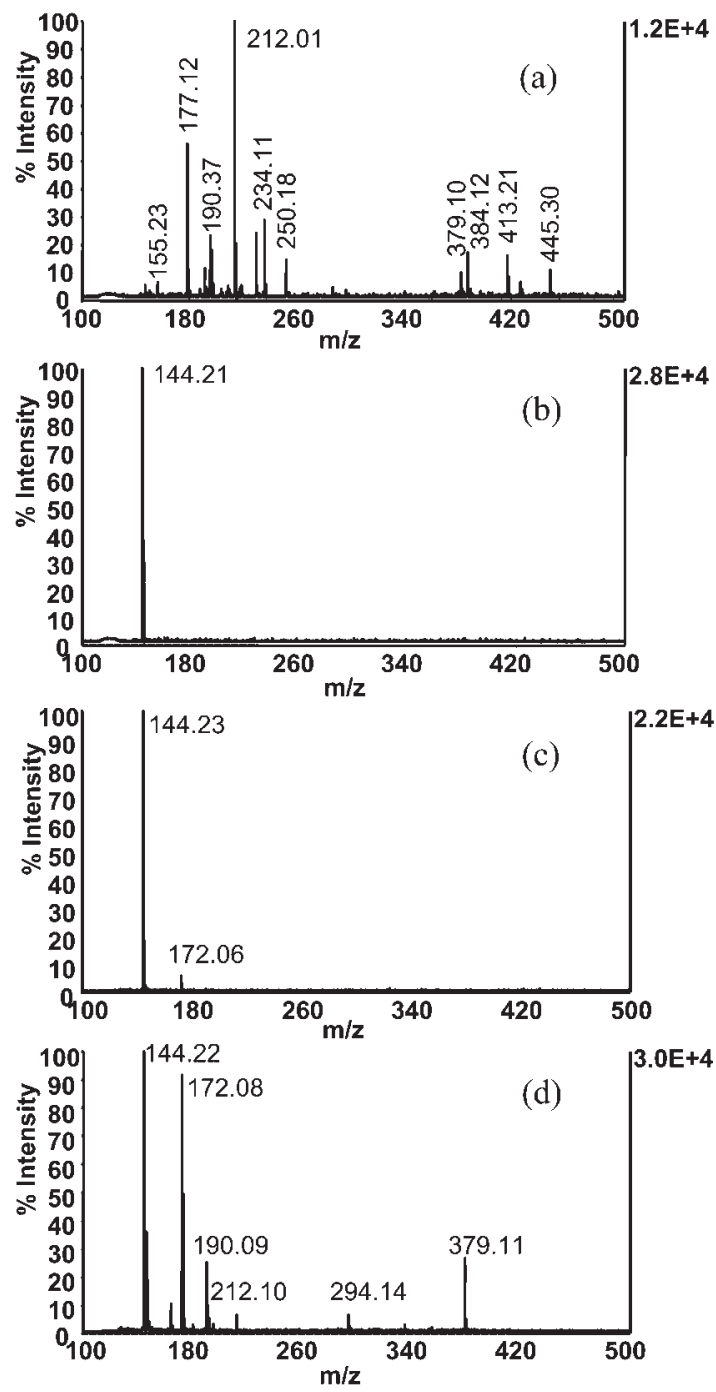

Figure 2. (a) MALDI mass spectrum of CHCA matrix only. Resulting spectra of CHCA matrix/HTAB surfactant at mole ratios of (b) 1000:1, (c) 1000:0.01, and (d) 1000:0.0001.

successfully suppressed. The abundant ion at $m / z 284$ is the surfactant-related ion $[\mathrm{CTAB}-\mathrm{Br}]^{+}$.

It can be seen, however, that the $p$-coumaric acid signals are also partially suppressed (Fig. 3(b)) resulting in a decrease in their net ion count. The average analyte ion signal intensity $(\mathrm{n}=5)$ at $m / z 147$ was $10127( \pm 14 \%)$ without surfactant, and decreased to $3949( \pm 11 \%)$ when surfactant

Table 1. Common CHCA fragment ions and adducts observed in mass spectra using a $337 \mathrm{~nm} \mathrm{~N}$ laser

\begin{tabular}{ll}
\hline$m / z$ & Ion form \\
\hline 122.08 & {$\left[\mathrm{M}+\mathrm{H}-\mathrm{C}_{3} \mathrm{H}_{2} \mathrm{NO}^{+}\right.$} \\
146.04 & {$\left[\mathrm{M}+\mathrm{H}-\mathrm{CN}-\mathrm{H}_{2} \mathrm{O}\right]^{+}$} \\
164.05 & {$[\mathrm{M}+\mathrm{H}-\mathrm{CN}]^{+}$} \\
172.04 & {$\left[\mathrm{M}+\mathrm{H}-\mathrm{H}_{2} \mathrm{O}\right]^{+}$} \\
190.05 & {$[\mathrm{M}+\mathrm{H}]^{+}$} \\
212.03 & {$[\mathrm{M}+\mathrm{Na}]^{+}$} \\
234.02 & {$\left[\mathrm{M}-\mathrm{H}+2 \mathrm{Na}^{+}\right.$} \\
294.07 & {$\left[2 \mathrm{M}+\mathrm{H}-\mathrm{CO}_{2}-\mathrm{C}_{2} \mathrm{H}_{3} \mathrm{~N}\right]^{+}$} \\
335.10 & {$\left[2 \mathrm{M}+\mathrm{H}-\mathrm{CO}_{2}\right]^{+}$} \\
379.09 & {$[2 \mathrm{M}+\mathrm{H}]^{+}$} \\
\hline
\end{tabular}




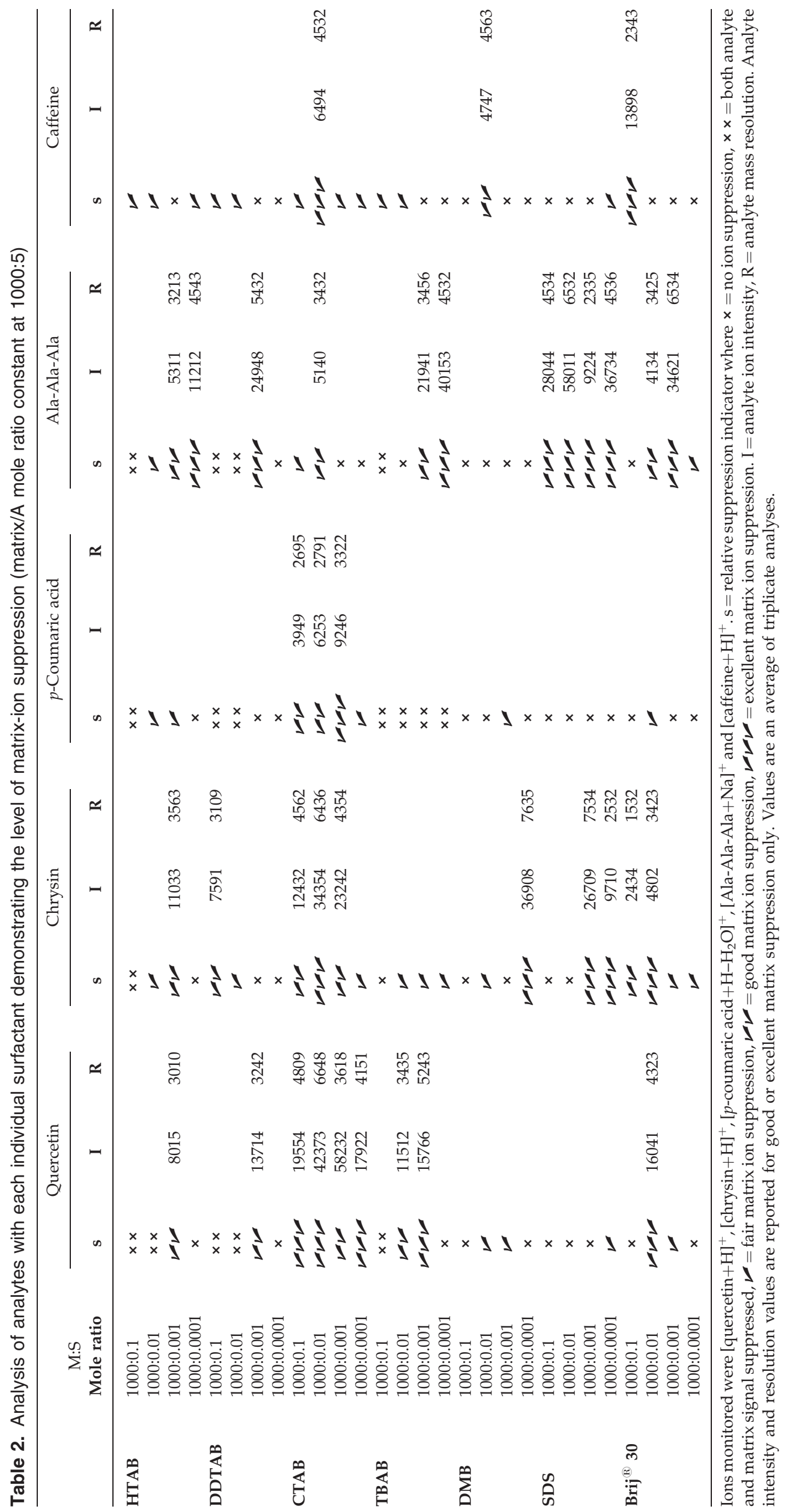



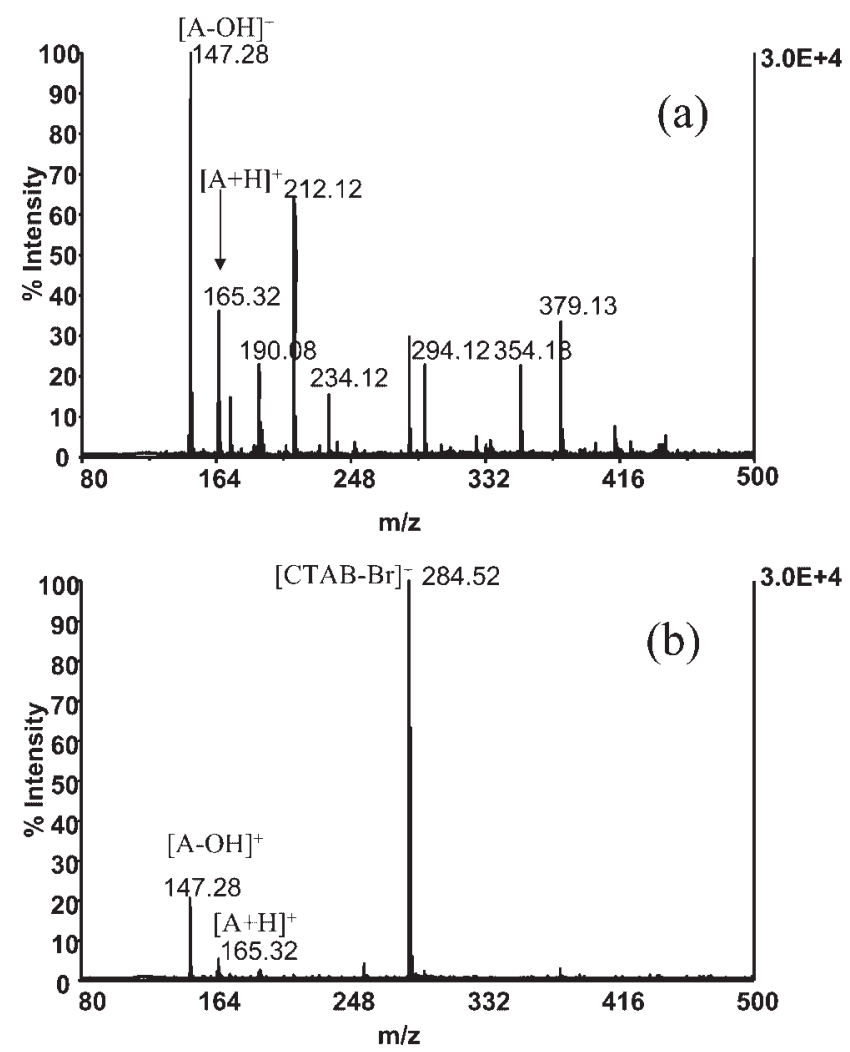

Figure 3. (a) MALDI mass spectrum of $\mathrm{CHCA} / \mathrm{p}$-coumaric acid (A) at mole ratio of 1000:5 and (b) mass spectrum of $\mathrm{CHCA} / p$-coumaric acid/CTAB at mole ratio of 1000:5:0.1.

was added. Despite the decrease in signal intensity, the resolution of the mass spectrum was noted to increase. Without surfactant, the calculated resolution was 2078 $( \pm 15 \%)$. The resolution increased to $2695( \pm 13 \%)$ when surfactant was used.

The matrix suppression effect from other surfactants at much lower surfactant concentration was also of interest. As an example, Fig. 4 shows the mass spectrum obtained when surfactant HTAB was used for the analysis of chrysin at a M/A/S mole ratio of 1000:5:0.001. This spectrum illustrates excellent matrix-ion suppression while still observing a

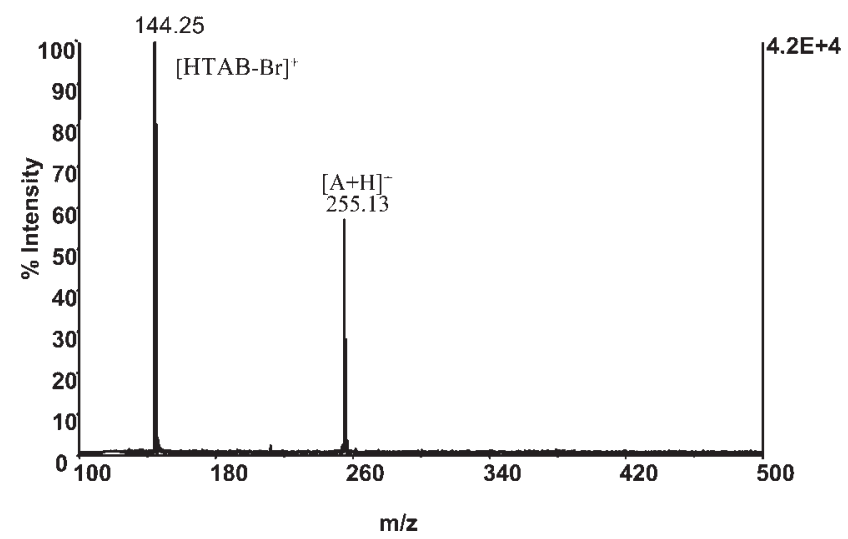

Figure 4. MALDI mass spectrum of $\mathrm{CHCA} /$ chrysin $(\mathrm{A}) / \mathrm{HTAB}$ at mole ratio of 1000:5:0.001.

Copyright (C) 2007 John Wiley \& Sons, Ltd.

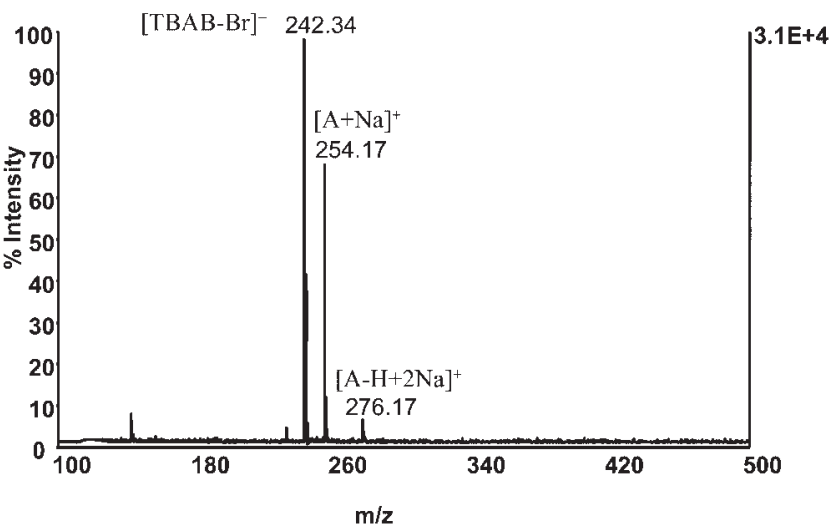

Figure 5. MALDI mass spectrum of $\mathrm{CHCA} /$ trialanine (A)/TBAB at mole ratio of 1000:5:0.001.

strong analyte signal even at very low surfactant concentration.

However, as seen in Table 2, only CTAB showed promise in matrix suppression for a wide range of analytes while still providing good analyte ion signal. The shorter hydrocarbon chain monocationic surfactants, DDTAB and HTAB, were useful at a low surfactant concentration, i.e. mole ratio of 1000:5:0.001, but only for the analysis of phenolic analytes. In future studies we would like to examine the effect of using a cationic quaternary ammonium surfactant with a longer hydrophobic tail than CTAB.

In addition to varying the hydrocarbon chain length of the quaternary ammonium surfactants, as was done in the series of $\mathrm{HTAB}$, DDTAB, and $\mathrm{CTAB}$, the other types of cationic surfactants, TBAB and $\mathrm{DMB}$, were also examined. TBAB contains four butyl groups attached to the ammonium ion and DMB has two cationic ammonium functionalities, one on either side of a ten-carbon-length chain. Only TBAB showed promising results, particularly for the analysis of the peptide trialanine (Table 2) and at low surfactant concentration. Figure 5 illustrates the result of mixing TBAB with $\mathrm{CHCA}$ for the analysis of trialanine (Ala-Ala-Ala). Matrix signal suppression was observed and the dominant ion at $\mathrm{m} / \mathrm{z}$ 242 corresponds to the surfactant ion $[\mathrm{TBAB}-\mathrm{Br}]^{+}$. The analyte signal is seen as a sodiated molecule $m / z 254$ and, to a lesser extent, a doubly sodiated ion $[\mathrm{A}+2 \mathrm{Na}-\mathrm{H}]^{+} \mathrm{m} / z 276$. The presence of the sodiated ions for trialanine in all MALDI spectra is because steps were not taken to lower sodium levels in the reagents. Also, our method employed 80:20 $\mathrm{MeOH} / \mathrm{H}_{2} \mathrm{O}$ as a solvent, rather than a mixture containing ACN and $0.1 \%$ TFA, normally used for the analysis of peptides. We maintained this solvent for simplicity, as it does not affect the $\mathrm{pH}$ of drying droplets, and it is comparable with other relevant studies. ${ }^{24,25}$

Finally, the anionic surfactant SDS and the neutral surfactant $\mathrm{Brij}^{\mathrm{R}} 30$ were examined as possible matrix-ion suppressors. Both were able to suppress CHCA matrix ions. Except for the analysis of trialanine, their use often led to unacceptable suppression of the analyte signal. Unlike the cationic surfactants, neither of these yielded surfactantrelated ions in either positive or negative ion mode. Figure 6(a) shows the mass spectrum obtained when SDS 


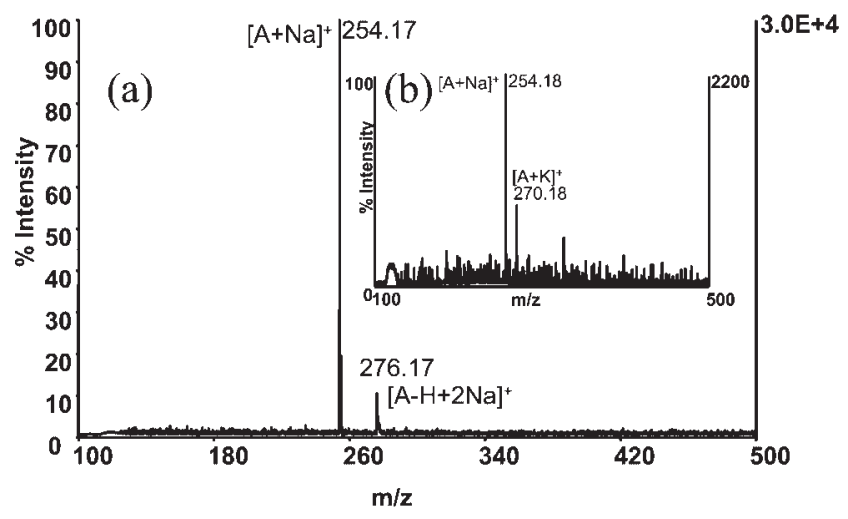

Figure 6. (a) MALDI mass spectrum of CHCA/trialanine (A)/ SDS at mole ratio of 1000:5:0.1, (b) MALDI mass spectrum of CHCA/trialanine (A)/SDS when analyte is diluted 1000-fold.

was used in the analysis of trialanine at a M/A/S mole ratio of 1000:5:0.1. Both the sodiated $(\mathrm{m} / \mathrm{z} 254)$ and the doubly sodiated adduct $(\mathrm{m} / \mathrm{z} 276)$ are observed with minimal background signals from the matrix. Figure 6(b) is the result of a 1000-fold dilution of the peptide analyte, while keeping the matrix and surfactant ratio constant (i.e. 1000:0.005:0.1). The sodiated and potassiated analyte ions are clearly observed with a $\mathrm{S} / \mathrm{N}$ ratio $>5$. Although this is a preliminary result, it does show that surfactant-mediated MALDI can be used for the analysis of low levels of small peptides.

Although the results shown in Table 2 are variable some general trends in surfactant performance can be stated. For the analysis of phenolics, CTAB showed, by far, the best performance for surfactant-mediated MALDI-TOFMS with strong analyte signals at nearly all surfactant concentrations. $\mathrm{CTAB}$ was the only surfactant suitable for the analysis of the phenolic acid, $p$-coumaric acid. This last result is surprising as this analyte is chemically very similar to the matrix CHCA but without the cyano group. For small peptide analysis, SDS was superior, consistently showing excellent matrix-ion suppression with strong analyte signals over a range of surfactant concentrations. This was not surprising as SDS is used commonly in the digests of peptides and proteins prior to analysis, as it helps to solubilize the analyte. Finally, the neutral surfactant, Brij ${ }^{\mathbb{R}} 30$, showed reasonable performance for the analysis of caffeine at relatively high surfactant concentration.

The resolution of the mass spectra for the best case scenarios typically ranges from 3000-5000, considered acceptable using this instrument. Resolution measurements with and without surfactant showed that in most cases resolution increased by $25-75 \%$ by using the most promising surfactant.

\section{Effect of concentration of surfactant}

In a previous ${ }^{24}$ and the present study, the mole ratio of matrix to surfactant was found to be an important factor in matrix-ion suppression while preserving the analyte signal. More detailed experiments were undertaken whereby the M/A mole ratio was maintained at 1000:5, while the amount of surfactant added was varied (1000:5:1 $\rightarrow 1 \times 10^{-5}$ ) to yield a surfactant concentration-dependence profile. The signal intensity of the major analyte ion was carefully monitored. Each combination of surfactant to analyte was tested and replicated five times for each concentration. As an example, Fig. 7 demonstrates the impact of changing the amount of Brij $^{\mathbb{R}} 30$ on the signal intensity of trialanine. When too much surfactant is added $(\mathrm{M} / \mathrm{S}=1000: 1)$, the analyte signal is absent because both the CHCA and analyte ions are fully suppressed. As the amount of added surfactant decreases, the analyte ion intensity increases to a maximum, then decreases at very low surfactant addition. Finally, as the amount of $\mathrm{Brij}^{\mathrm{R}} \quad 30$ becomes negligible $(\mathrm{M} / \mathrm{A} / \mathrm{S}=$ 1000:5:0.00001), the analyte signal increases again. This is because there is little or no suppression, and the resulting spectrum is comparable with those of the matrix and the analyte alone. Each point on the graph was averaged for five identical spots and relative standard deviation (RSD) values ranged from 7 to $22 \%$. These RSD values are typical of MALDI experiments and compare with those reported in the literature. ${ }^{26-28}$

Generalizations regarding optimal surfactant loadings were difficult because the results were both analyte- and surfactant-dependent. On the whole, M/S mole ratios of 1000:0.01 and 1000:001 provided the best results. If one has an unknown analyte, an initial M/A/S mole ratio of 1000:5:0.01 is a good starting point for analysis and CTAB should be used first.

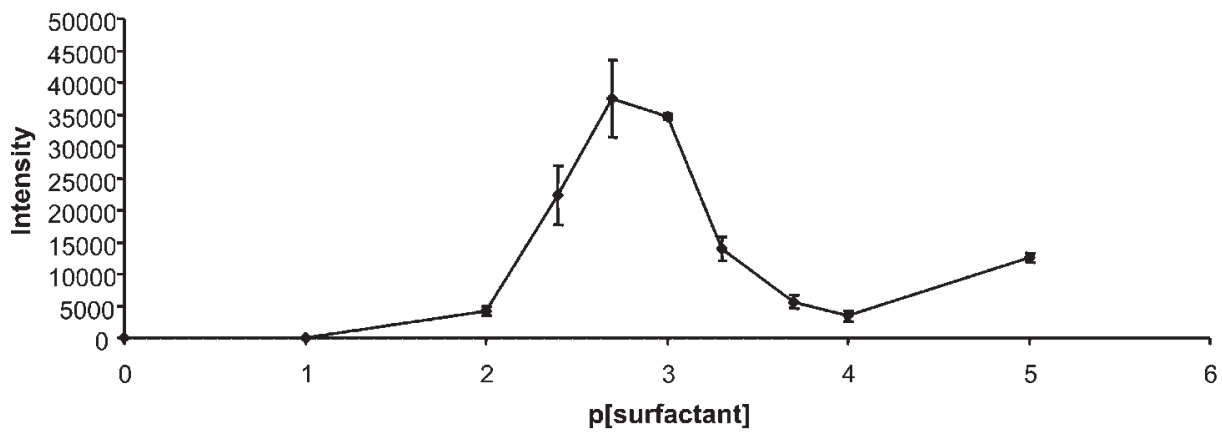

Figure 7. Molar concentration profile displaying the effect of $\mathrm{Brij}^{\circledR} 30$ concentration on $[\text { trialanine }+\mathrm{Na}]^{+}, \mathrm{m} / \mathrm{z} 254$ ion. The mole ratio of $\mathrm{CHCA} /$ trialanine was held constant at 1000:5 $(n=5)$. 

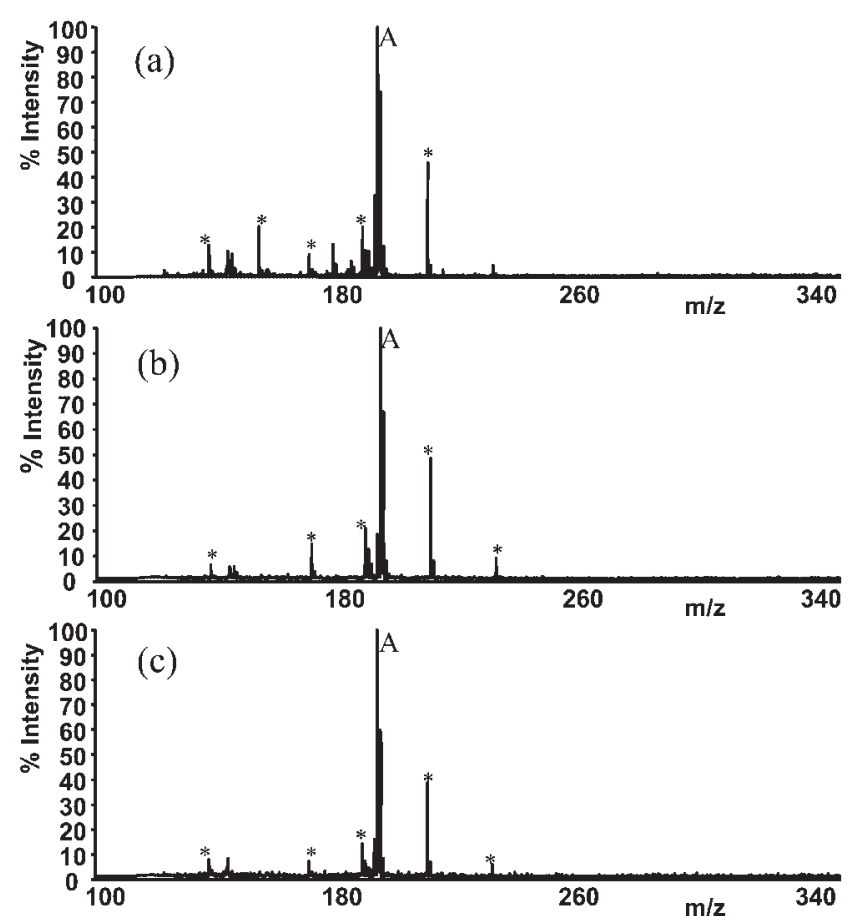

Figure 8. Monitoring the change in MALDI mass spectra of $\mathrm{CHCA}$ /caffeine (mole ratio 1000:5) as number of laser shots varied; (a) 5 shots, (b) 10 shots, (c) 30 shots. $A=$ analyte; ${ }^{*}=$ matrix ions.

\section{Heterogeneity of surfactant-containing sample spots}

One desired feature of MALDI sample preparation is that a given spot should be homogeneous. However, we believe that spots containing added surfactant may lead to a heterogeneous sample. The use of MALDI-TOFMS to examine sample spots containing heterogeneous layers has been investigated recently. ${ }^{29,30}$ Hanton et al. demonstrated this by using electrospray deposition to create a two-layered sample of poly(methyl methacrylate) (PMMA) and polystyrene (PS). ${ }^{29}$ Controlling the number of laser shots used during analysis led to successful depth-profiling experiments where these polymers could be independently identified. Recently, the dried-droplet spotting approach for poly(oxyethylene) and poly(oxypropylene) triblock copolymers was tested. ${ }^{30}$ Changing the number of laser shots from 50 to 300 in this sample resulted in the emergence of a new ion distribution, particularly in the low-mass range. This was due to the presence of multiple layers within the deposit, with lighter products located near the core and heavier products spreading out on the surface of the drop.

The same approach to depth profiling was undertaken in this study. As a preliminary experiment caffeine was chosen as a model analyte and CTAB as the surfactant. Figure 8 represents the mass spectral results with increasing laser shots (5, 10 and 30 shots) on caffeine/CHCA matrix only. The intensities of analyte signal (A) and matrix ions $\left(^{*}\right)$ remain relatively unchanged with increased laser shots, suggesting that these crystallized samples are fairly homogeneous.

Figure 9 shows the effect of increased laser shots on the MALDI spectra of a matix/analyte/surfactant sample with a

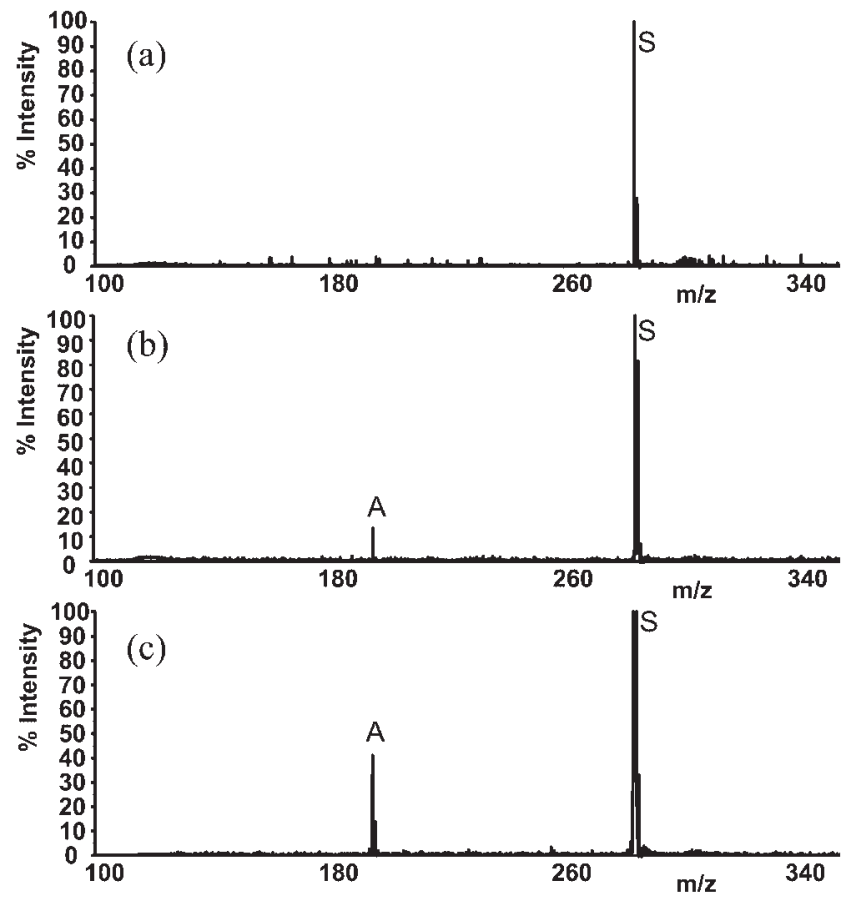

Figure 9. Monitoring the change in MALDI mass spectra of CHCA/caffeine/CTAB (mole ratio 1000:5:1) as number of laser shots varied; (a) 5 shots, (b) 10 shots, (c) 30 shots. $\mathrm{A}=$ analyte; $\mathrm{S}=$ surfactant.

M/A/S mole ratio of 1000:5:1. With only five laser shots, the spectrum in Fig. 9(a) reveals that suppression of both analyte and matrix-related ions has occurred. The surfactant ion is the only one present in the spectrum. However, as the laser shots increase to 10 and then to 30 , a partially suppressed analyte signal (A) is observed. Meanwhile the matrix-related ions remain totally suppressed. These results suggest that the surfactant concentration is too high and that strong ion suppression is occurring, particularly near to the surface of the sample.

A further dilution of the surfactant concentration (M/A/ $S=1000: 5: 0.1)$ reveals a somewhat different spectral pattern (Fig. 10). When five laser shots are employed, the surfactant and analyte ions are both readily observed. When ten laser shots are used, the analyte and surfactant ions are again observed along with two identifiable matrix-related ions. As the number of laser shots is increased to 30 , the matrix ions become more apparent and the surfactant signal decreases in intensity. Since the surfactant signal is strong after five and ten laser shots but not at 30 shots, this again suggests that the surfactant it is a major component near the surface. That is, samples containing surfactant do not crystallize to a homogeneous spot, rather these spots are more heterogeneous with higher amounts of surfactant near the surface.

As we were aware that the MALDI spectra discussed above represent only single analysis and that variations from spot to spot could occur, a more detailed experiment was undertaken whereby ion counts of selected ions from matrix, surfactant and analyte ionization were measured as the number of laser shots was increased from 5 to 50, in increments of 5 . The ions at $\mathrm{m} / \mathrm{z} 195,212$, and 284 were 


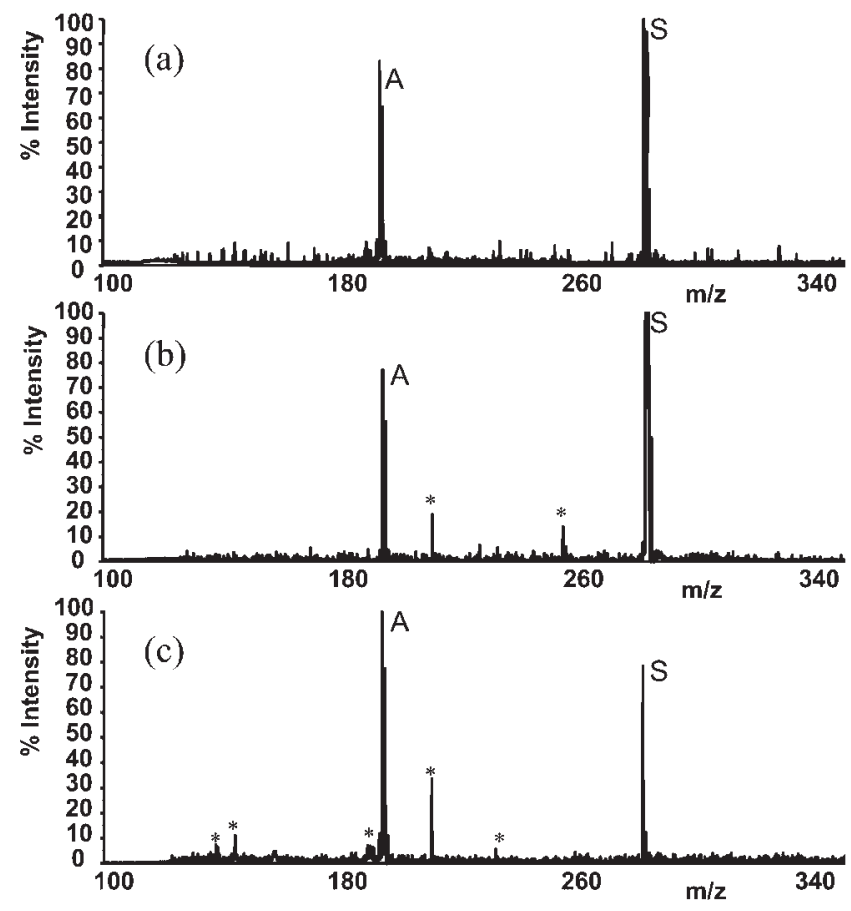

Figure 10. Monitoring the change in MALDI mass spectra of CHCA/caffeine/CTAB (mole ratio 1000:5:0.1) as number of laser shots varied; (a) 5 shots, (b) 10 shots, (c) 30 shots. $\mathrm{A}=$ analyte; $\mathrm{S}=$ surfactant; $^{*}=$ matrix ions.

monitored for the presence of caffeine $[\mathrm{A}+\mathrm{H}]^{+}$, matrix $[\mathrm{CHCA}+\mathrm{Na}]^{+}$and surfactant $[\mathrm{CTAB}-\mathrm{Br}]^{+}$, respectively. The results of depth profiling of replicate spots containing matrix and analyte only are shown in Fig. 11(a). The ion intensity profiles of the analyte and matrix signals follow closely together, indicating spot homogeneity. It should be noted that the results in Fig. 11 are average ion counts from ten replicate spots where RSDs range from 6-10\%. The ion intensity recorded is the average ion count per laser shot. As can be seen in the graph, the intensity of the ion counts/shot began to decrease after approximately 30 laser shots, indicating that less sample remains after each subsequent laser shot and that the material is depleted.

When CTAB was added so that the $\mathrm{M} / \mathrm{A} / \mathrm{S}$ mole ratio was 1000:5:1 the [CTAB-Br] $^{+}$ion was the dominant ion in the mass spectra, as expected. At this $\mathrm{M} / \mathrm{A} / \mathrm{S}$ ratio, CHCA-related ions were totally suppressed. The caffeine signal is observable, but it is partially suppressed by the surfactant. As the number of laser shots is increased to 20, the surfactant signal decreases by half indicating a higher concentration of surfactant is nearer the surface of the drop.

Lowering the CTAB concentration so that the $\mathrm{M} / \mathrm{A} / \mathrm{S}$ ratio is now 1000:5:0.1 showed an even more intriguing result, particularly in the region of lower number of laser shots. Essentially, at the top of the droplet (ca. 5 laser shots) the caffeine signal is very intense and equal in intensity to that of the surfactant-related ion. In contrast, the matrix-related ion is of minor intensity. This indicates that surfactant and analyte concentrations near the upper layer of the spots were
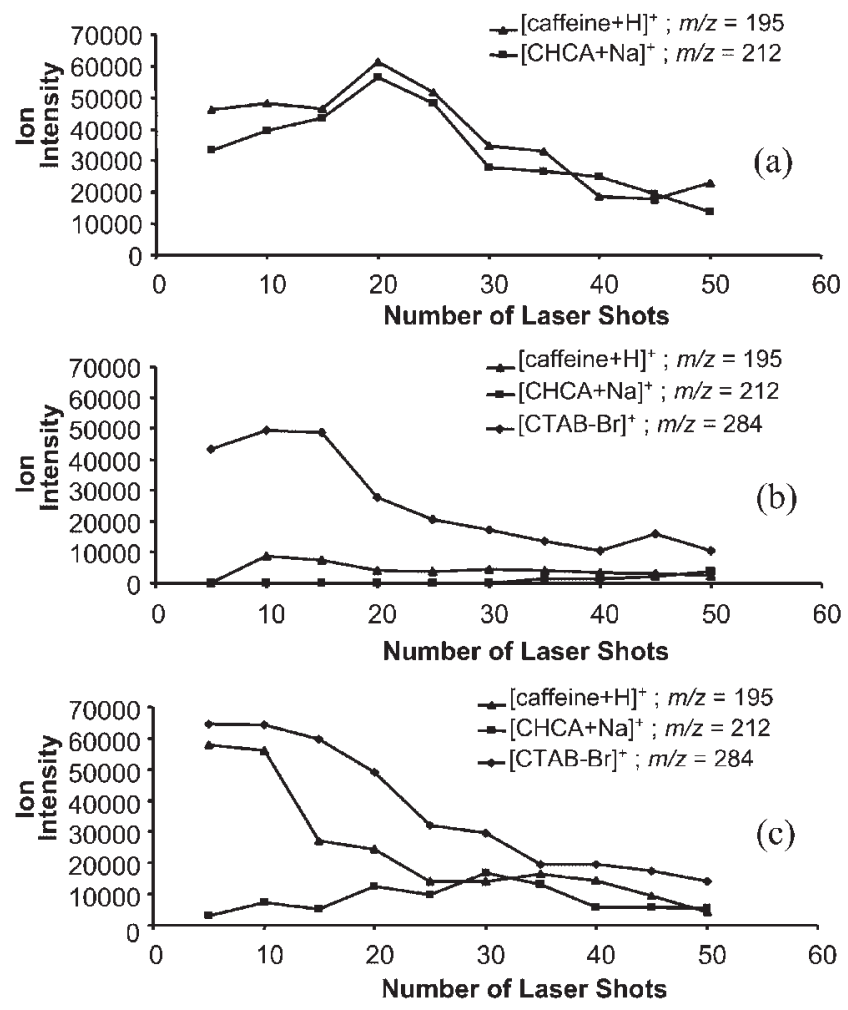

Figure 11. Monitoring the change in average ion signal intensity of selected ions as the number of laser shots is increased on individual spots $(n=10)$ : ion profiles of (a) CHCA/caffeine, (b) $\mathrm{CHCA} /$ caffeine/CTAB where mole ratio is $1000: 5: 1$, and (c) $\mathrm{CHCA} /$ caffeine/CTAB where mole ratio is 1000:5:0.1. RSD values range from 6 to $10 \%$.

ideal for surfactant-mediated MALDI. Interestingly, as soon as the number of laser shots was increased, the CTAB and caffeine signals suddenly dropped, suggesting a decrease in both their concentrations with depth. On the other hand, the matrix ion increased steadily suggesting that less and less surfactant is present to suppress the matrix.

From the above experiments it is proposed that, during the crystallization process, the surfactant tends to migrate towards the top of the droplet. Surfactants, in particular cationic surfactants, may be able to attract analyte molecules to the top of a droplet. It is likely that at this point, the mole ratio of matrix/analyte is much lower than that expected by the initial mixing ratios. Thus, similar to the reported mechanism of the mole ratio dependent matrix suppression effect (MSE), ${ }^{21}$ the matrix-matrix interactions are minimized and each analyte molecule is surrounded by just enough matrix to absorb the laser energy and ionize it. It is also possible that micelles may form in the top region of the spot as long as there is enough surfactant added initially and this may have an effect on matrix ion suppression.

Further depth-profiling experiments on other types of surfactants and analytes, and the use of spectroscopic surface analysis methods (i.e. scanning electron microscopy), are required before the surfactant-mediated MALDI mechanism can be fully understood. 


\section{CONCLUSIONS}

Surfactant-enhanced MALDI is a useful technique for the analysis of various classes of small molecules. We have tested several cationic surfactants, all bromide salts, as well as SDS and a neutral surfactant, Brij ${ }^{\circledR}$ 30. Suppression of positive CHCA matrix-related ions can be achieved, along with increased analyte resolution. The detection limit for the peptide trialanine was found to be in the 100 femtomole range. It seems that the surfactant is enriched near the surface of the spot along with the analyte and that this enrichment lessens the production of interfering matrix-related ions. Surfactant-mediated MALDI will be further explored for use in the identification of small biomolecules and analyte mixtures with the aid of MALDI-MS/MS.

\section{Acknowledgements}

The Department of Chemistry and the School of Graduate Studies (Memorial University) and NSERC are thanked for their financial support. Technical support from the Center for Chemical Analysis and Research Training (C-CART) is appreciated.

\section{REFERENCES}

1. Karas M, Hillenkamp F. Anal. Chem. 1988; 60: 2299.

2. Tanaka K, Waki H, Ido Y, Akita S, Yoshida Y, Yoshida T. Rapid Commun. Mass Spectrom. 1988; 2: 151.

3. Karas M, Bahr U, Ingendoh A, Nordhoff E, Stahl B, Strupat K, Hillenkamp F. Anal. Chim. Acta 1990; 241: 175.

4. Distler AM, Allison J. Anal. Chem. 2001; 73: 5000.

5. Bahr U, Deppe A, Karas M, Hillenkamp F. Anal. Chem. 1992; 64: 2866.

6. Weidner S, Kuhn G, Friedrich J. Rapid Commun. Mass Spectrom. 1998; 12: 1373.

7. Michalak L, Fisher K, Alderdice D, Jardine D, Willett G. Org. Mass Spectrom. 1994; 29: 512.

8. Cornett DS, Amster IJ, Duncan MA, Rao AM, Eklund PCJ. J. Phys. Chem. 1993; 97: 5036.

9. Lai EPC, Owega S, Kulczycki R. J. Mass Spectrom. 1998; 33: 554.

10. Zhang Q, Zou H, Gou Z, Zhang Q, Chen X, Ni J. Rapid Commun. Mass Spectrom. 2001; 15: 217.

11. Schurenberg M, Dreisewerd K, Hillenkamp F. Anal. Chem. 1999; 71: 221.

12. Kinumi T, Saisu T, Takayama M, Niwa H. J. Mass Spectrom. 2000; 35: 417.

13. Ayorinde FO, Hambright P, Porter TN, Keith QL. Rapid Commun. Mass Spectrom. 1999; 13: 2474.

14. Ayorinde FO, Garvin K, Saeed K. Rapid Commun. Mass Spectrom. 2000; 14: 608.

15. Xu S, Li Y, Qiu J, Guo Z, Guo B. Anal. Chem. 2003; 75: 6191.

16. Najam-ul-Haq M, Rainer M, Schwarzenauer T, Huch CW, Bonn GK. Anal. Chim. Acta 2006; 561: 32.

17. Hu L, Xu S, Pan C, Yuan C, Zou H, Jiang G. Environ. Sci. Technol. 2005; 39: 8442.

18. Pan C, Xu S, Hu L, Su X, Ou J, Zou H, Guo Z, Zhang Y, Guo B. J. Am. Soc. Mass Spectrom. 2005; 16: 883.

19. Ren S, Zhang L, Chen Z, Guo Y. J. Am. Soc. Mass Spectrom. 2005; 16: 333.

20. Chan TWD, Colburn AW, Derrick PJ. Org. Mass Spectrom. 1991; 26: 342.

21. Knochenmuss R, Dubois F, Dale MJ, Zenobi R. Rapid Commun. Mass Spectrom. 1996; 10: 871.

22. McCombie G, Knochenmuss R. Anal. Chem. 2004; 76: 4990.

23. Knochenmuss R, Karbach V, Wiesli U, Breuker K, Zenobi R. Rapid Commun. Mass Spectrom. 1998; 12: 529.

24. Guo Z, Zhang Q, Zou H, Guo B, Ni J. Anal. Chem. 2002; 74: 1637.

25. Su AK, Liu JT, Lin CH. Talanta 2005; 67: 718.

26. Sleno L, Volmer DA. Rapid Commun. Mass Spectrom. 2006; 20: 1517.

27. Dekker LJ, Dalebout JC, Siccama I, Jenster G, Smitt PAS, Luider TM. Rapid Commun. Mass Spectrom. 2005; 19: 865.

28. Kang MJ, Pyun JC, Lee JC, Choi YJ, Park JH, Lee JG, Choi HJ. Rapid Commun. Mass Spectrom. 2005; 19: 3166.

29. Hanton SD, Hyder IZ, Stets JR, Owens KG, Blair WR, Guttman CM, Giuseppetti AA. J. Am. Soc. Mass Spectrom. 2003; 15: 168.

30. Terrier P, Buchmann W, Cheguillaume G, Desmazieres B, Tortajada J. Anal. Chem. 2005; 77: 3292. 